\title{
Molecular Identity of Dendritic Voltage-Gated Sodium Channels
}

\author{
Andrea Lorincz ${ }^{*}$ and Zoltan Nusser ${ }^{*}$ \\ Laboratory of Cellular Neurophysiology, Institute of Experimental Medicine, Hungarian Academy \\ of Sciences, 1083 Budapest, Hungary
}

\begin{abstract}
Active invasion of the dendritic tree by action potentials (APs) generated in the axon is essential for associative synaptic plasticity and neuronal ensemble formation. In cortical pyramidal cells (PCs) this AP backpropagation is supported by dendritic voltage-gated $\mathrm{Na}^{+}$channels (Nav), whose molecular identity is unknown. Using a highly sensitive electron microscopic immunogold technique, we reveal the presence of the Nav1.6 subunit in hippocampal CA1 PC proximal and distal dendrites. Here the subunit density is lower by a factor of 35 to 80 than that found in axon initial segments. A gradual decrease in Nav1.6 density along the proximodistal axis of the dendritic tree was also detected without any labeling in dendritic spines. Our results reveal the characteristic subcellular distribution of the Nav1.6 subunit, identifying this molecule as a key substrate enabling dendritic excitability.
\end{abstract}

Associative synaptic plasticity in cortical pyramidal cells (PC) is the most widely accepted cellular model of learning. An essential prerequisite of the model is that input synapses, which are distributed over an enormously large dendritic tree, must be capable of sensing the precise timing of the output signal. The most likely mechanism for this is the active invasion of the dendritic tree by fast sodium action potentials (APs) initiated in the axon initial segment (AIS). Modification of backpropagating APs therefore holds tremendous potential for altering synaptic plasticity and the formation of neuronal representations. Voltage-gated $\mathrm{Na}^{+}$(Nav) currents have been detected in hippocampal and neocortical PC dendrites, where they not only support AP backpropagation, but also underlie nonlinear synaptic integration and dendritic sodium spike generation (1-6; reviewed by 7-10). Patch-clamp experiments demonstrate that axonal and somato-dendritic Nav currents differ in their activation and inactivation properties $(11,12)$, indicating either different Nav subunit compositions or distinct posttranslational modifications of identical subunits (12-14). However, the lack of subunit-specific drugs precludes the unequivocal identification of the subunit composition of the axo-somato-dendritic Nav channels using functional approaches. Immunohistochemistry with subunit-specific antibodies offers an alternative experimental approach to address this issue. Nav1.1, Nav1.2 and Nav1.6 are the Nav subunits expressed in adult brains $(15,16)$. They have been detected in nodes of Ranvier, AISs and preterminal axons using immunofluorescence localization (17-22). Unfortunately this method has so far failed to visualize any of these Nav subunits in somato-dendritic compartments, most likely due to its insufficient sensitivity. We modified the immunofluorescence technique to increase its sensitivity and employed a highly sensitive, quantitative electron microscopic (EM) immunogold method (SDS-digested freeze-fracture replica-labeling: SDS-FRL, 23-25) to identify the Nav subunit content of the somato-dendritic plasma membranes of CA1 PCs.

In conventional immunofluorescence localization techniques, the tissue is usually fixed with a phosphate buffered fixative containing paraformaldehyde, resulting in a fixation that

`Correspondence should be addressed either to nusser@koki.hu or to lorincz@koki.hu. 
masks antigens in many subcellular compartments, including e.g. postsynaptic densities and AISs (26). The visualization of antigens in some of these compartments might be possible following antigen-retrieval, such as microwave irradiation or pepsin digestion $(27,28)$. We employed a low-pH based fixation approach (Supporting Online Material) that vastly increased the strength of the reactions for almost all $\mathrm{GABA}_{\mathrm{A}}$ and glutamate receptors, and voltage-gated $\mathrm{K}^{+}$and $\mathrm{Na}^{+}$channels, and allowed the visualization of these proteins in postsynaptic membranes and AISs without antigen retrieval. Using this approach, we first employed pan-Nav antibodies to visualize all Nav subunits in the hippocampal CA1 area of the adult rat. Immunoreactions with pan-Nav antibodies revealed a very strong labeling of AISs and nodes of Ranvier in stratum pyramidale (SP) overlying a rather homogeneous staining of the strata oriens (SO), radiatum (SR) and lacunosum-moleculare (SLM; Fig. 1A). The increased sensitivity afforded by the low-pH fixation condition allowed the visualization of pan-Nav immunosignal associated with somatic and proximal dendritic plasma membranes of PCs (Fig. 1B) that were also outlined by strong Kv2.1 subunit immunoreactivity. The intensity of the dendritic pan-Nav labeling was much weaker than that of the AISs, consistent with functional predictions (29). Interestingly, in some PCs, strongly pan-Nav labeled AISs originated from the apical dendrites and a stronger pan-Nav labeling was associated with the plasma membrane of these dendrites around the origin of the AIS (fig. S1). Our pan-Nav immunofluorescence reactions visualized Nav channels in the somato-dendritic compartments of PCs and revealed large differences in Nav density between AISs and somato-dendritic plasma membranes. However, A) the subunit composition, B) the presence or absence of Nav channels in small dendritic compartments such as small oblique dendrites and dendritic spines and C) the exact quantitative difference in Nav density between distinct subcellular compartments remained elusive.

To address the first of these issues, we carried out immunofluorescence reactions with specific antibodies against the Nav1.1, Nav1.2 and Nav1.6 subunits. After confirming the specificity of the immunoreactions (fig. S2, S3A), we investigated their distributions in the CA1 area. The labeling pattern obtained with the pan-Nav antibodies was essentially the sum of the patterns obtained for these three subunits (Fig. 1A). Immunosignal for the Nav1.1 subunit was restricted to small axonlike profiles in the SP, diffuse signal for the Nav1.2 subunit was observed in the neuropil of the SO, SR and SLM, whereas intense Nav1.6 labeling was confined to AISs and nodes of Ranvier (Fig. 1A). Double immunolabeling for Kv3.1b and Nav1.1 subunits demonstrated that this Nav subunit is present in small axonlike processes and AISs of Kv3.1b immunopositive GABAergic interneurons (fig. S3), but not in PCs, in agreement with a previous study (19). Double immunofluorescence reactions revealed the presence of the Nav1.2 subunit in the proximal part of PC AISs (Fig. 2A; 17), but no Nav1.2 subunit immunoreactivity could be detected in the plasma membranes of somata and proximal apical dendrites. The diffuse Nav1.2 subunit immunolabeling of the neuropil was associated with vGluT1 immunopositive presynaptic axons (Fig. 2B), but not with Kv4.2 immunoreactive dendritic shafts or spines of CA1 PCs (Fig. 2C), demonstrating the predominantly presynaptic expression of this subunit. Very intensely labeled AISs and nodes of Ranvier dominated the pictures obtained for the Nav1.6 subunit. In addition, in our best reactions, very weak plasma membrane-like labeling of PC apical dendrites could be observed (Fig. 2D, E). This labeling decreased in intensity when individual dendrites were followed from their somata towards their apical tips (Fig. 1A). Weak dendritic Nav1.6 labeling could be detected in those PCs in which AISs originated from the apical dendrites (Fig. 2F). However, even in our best reactions, small diameter oblique apical and basal dendrites, as well as dendritic spines remained immunonegative. These results provide molecular identification of dendritic Nav channels and predict complex subcellular compartment-and distance-dependent regulation of the cell-surface density of Nav1.6 in PC dendritic trees. 
To identify the potential presence of the Nav1.6 subunit in small dendritic compartments and to quantify the differences in the plasma membrane density of Nav1.6 in distinct subcellular compartments, we adopted SDS-FRL $(23,24)$, a recently developed quantitative EM immunolocalization method with exceptionally high sensitivity. The labeling efficiency for synaptic AMPA receptors of $\sim 100 \%$ using SDS-FRL (30) is approximately 2.5 -fold higher than that achieved with a postembedding immunogold localization of the same subunits with an identical antibody (31). A labeling efficiency of $100 \%$ means that, on average, every protein is represented by a gold particle with a localization method that has a spatial resolution of $\sim 25 \mathrm{~nm}$. We therefore exploited the potential of this method for Nav1.6 localization by preparing carbon-platinum-carbon replicas of the CA1 area following highpressure freezing and freeze-fracturing, and immunoreacted them with our anti-Nav1.6 antibodies (18). First we sought to examine the immunogold content of AISs (Fig. 3), the most intensely labeled subcellular structures found in the immunofluorescence reactions. Many elongated protoplasmic face (P-face) membrane structures in SP and SO contained a very high density of gold particles (Fig. 3A). These structures were then molecularly identified as AISs in double labeling experiments with AIS markers such as panNeurofascin and Ankyrin-G (Fig. 3B-D). The labeling on the P-face membranes is consistent with the cytoplasmic location of the epitope recognized by our antibody (intracellular loop between domains II and III) given the accepted transmembrane topology of the Nav1.6 subunit. In a few cases, AISs were fractured together with a fragment of the corresponding somatic plasma membrane (Fig. 3H), allowing us to assess how Nav1.6 density changes in the AIS as a function of distance from the soma. The graded increase in the density of the Nav1.6 subunit along the proximo-distal axis of PC AISs observed in the immunofluorescence reactions (Fig. 3G) could be also captured in replicas (Fig. 3H-L). Furthermore, gold particles labeling the Nav1.6 subunit on AISs consistently avoided the perisynaptic and synaptic membrane areas of axo-axonic GABAergic synapses, identified by clusters of intramembrane particles (Fig. 3A, fig. S4B), or by intense clustering of gold particles labeling neuroligin-2 (fig. S4C) or $\mathrm{GABA}_{\mathrm{A}}$ receptor $\beta 3$ subunit (fig. $\mathrm{S} 4 \mathrm{D}$, E). The absence of the Nav1.6 subunit in GABAergic axo-axonic synapses is another feature that is reliably captured with SDS-FRL, as well as with double immunofluorescence labeling for Nav1.6 and e.g. the $\mathrm{GABA}_{\mathrm{A}}$ receptor a 2 subunit (fig. S4A). Large somatic P-face membrane structures in SP contained many more gold particles for the Nav1.6 subunit than membranes fractured to the extracellular face (E-face; Fig. 4A). A similar picture was observed on proximal apical dendrites, where P-face dendritic shaft membranes contained gold particles (Fig. 4B, C), consistent with our fluorescent observations. Following the successful visualization of high density Nav1.6 labeling in AISs and a much lower density in somata and proximal apical dendrites, we turned our attention to small dendritic compartments, which remained immunonegative even in our best fluorescence reactions. Dendritic shafts and spines of apical oblique dendrites were sampled at proximal $(25-50 \mu \mathrm{m}$ from SP) and distal (100-150 $\mu \mathrm{m}$ ) locations in the SR, as well as dendritic shafts in SLM. Gold particles were observed on the P-face of dendritic shaft membranes in all locations, but dendritic spines were consistently immunonegative (Fig. 4C, D). An intrinsic property of immunogold localization is that gold particles can be found in any location by chance. Therefore, the presence of Nav1.6 subunits cannot be concluded just from the presence of a few gold particles in a given compartment, but requires a quantitative assessment of whether the particle density in a given location is significantly higher than that expected from a random process. We determined the nonspecific background labeling within our reactions by counting gold particles on the E-face plasma membranes (where the intracellular epitope of Nav subunits is not present) and found that on average $1.2 \pm 0.4$ gold particles ( $\mathrm{n}=5$ rats) are expected to be present in every $\mu \mathrm{m}^{2}$ membrane by chance (fig. S5). We then determined the density of gold particles labeling the Nav1.6 subunit on P-face plasma membranes of somata $\left(6.3 \pm 1.8 \mathrm{gold} / \mu \mathrm{m}^{2}\right.$ (mean $\left.\left.\pm \mathrm{SD}\right), \mathrm{n}=5 \mathrm{rats}\right)$, proximal apical dendrites $(6.5 \pm 1.5$ $\left.\mathrm{gold} / \mu \mathrm{m}^{2}\right)$, proximal oblique dendrites $\left(4.6 \pm 1.4 \mathrm{gold} / \mu \mathrm{m}^{2}\right)$, distal apical dendrites $(3.5 \pm$ 
$\left.1.4 \mathrm{gold} / \mu \mathrm{m}^{2}\right)$, distal oblique dendrites $\left(3.0 \pm 1.7 \mathrm{gold} / \mu \mathrm{m}^{2}\right)$ and dendrites in SLM $(2.1 \pm$ $0.6 \mathrm{gold} / \mu \mathrm{m}^{2}$ ). Of these, somata, proximal apical, proximal oblique and distal apical dendrites displayed a significantly higher density of gold particles than the background labeling (Fig. 4B, One-way ANOVA with Dunnett's post-hoc test, $\mathrm{p}<0.05$ ). Thus, the exceptionally high sensitivity of the SDS-FRL method allowed us to reveal low, but significant densities of the Nav1.6 subunit in these dendritic compartments, which remained immunonegative with the immunofluorescence method. Along these lines, it should be noted that the presence of low densities of other Nav subunits (e.g. Nav1.2) in somato-dendritic compartments cannot be excluded based on immunofluorescence data only, but highly sensitive reactions must be accomplished with SDS-FRL in order to unequivocally determine their presence or absence in identified subcellular compartments. Finally, we compared the Nav1.6 subunit density in somato-dendritic compartments to that found in AISs and nodes of Ranvier. Nodes of Ranvier in the alveus were very strongly immunolabeled for the Nav1.6 subunit (Fig. 3E, F), containing immunogold particles at almost twice the density as that observed in AISs $\left(346.3 \pm 82.9\right.$ vs. $\left.186.9 \pm 29.9 \mathrm{gold} / \mu^{2}{ }^{2}\right)$. The AISs contained $39 \pm 9$ - and $36 \pm 6$-times higher densities of gold particles than somata and proximal apical dendrites, respectively. These ratios are very similar to those estimated (29) for neocortical layer 5 PCs using whole-cell Nav current recordings ( $\sim 5$ fold) and $\mathrm{Na}^{+}$ imaging ( 30 fold). When dendritic compartments at the same distance from the soma were compared, we found that oblique dendrites contain less Nav1.6 subunit than main apical dendrites. There was also a clear tendency for a distance-dependent reduction in the density of gold particles along the proximo-distal axis of the dendritic tree (Fig. 4B). We could not detect immunogold particles labeling the Nav1.6 subunit above the background level in preterminal axons, axon terminals or dendritic spines.

Using an improved immunofluorescence method and SDS-FRL, we identify Nav1.6 as the prominent somato-dendritic Nav channel subunit in hippocampal PCs. Using SDS-FRL, we demonstrate a unique subcellular distribution pattern of the Nav1.6 subunit on the axosomato-dendritic compartments of PCs, revealing a very dramatic drop (40-70-fold) in density from nodes of Ranvier and AISs to somata, and a distance-dependent decrease in density along the proximo-distal axis of the dendritic tree. By exploiting the exquisite sensitivity of the SDS-FRL method, we obtained a gold particle density for the Nav1.6 subunit of $187 \pm 30\left(\mathrm{gold} / \mu \mathrm{m}^{2}\right)$ in AISs of CA1 PCs. A previous study (29) using in vitro electrophysiology, $\mathrm{Na}^{+}$imaging and multicompartmental modeling estimated a conductance density of Nav channels in neocortical layer 5 PC AISs of $2500 \mathrm{pS} / \mu \mathrm{m}^{2}$; given a $17 \mathrm{pS}$ single-channel conductance this predicts a functional channel density of $\sim 150$ channels per $\mu \mathrm{m}^{2}$. If CA1 PCs have an AIS Nav channel density similar to layer 5 PCs, we can conclude that the labeling efficiency of our SDS-FRL method is close to $100 \%$, similar to that obtained for synaptic AMPA receptors (30). Given this estimated labeling efficiency, our results predict a Nav channel density (channels per $\mu \mathrm{m}^{2}$ plasma membrane) of 5 in somata and proximal apical dendrites, 3 in proximal oblique dendrites and 2 in distal apical dendrites. The differences in the density of Nav1.6 in somatic and dendritic compartments represent only one level of complexity. Additional compartment-dependent variations in e.g. the phosphorylation states of the channels (12) could further increase functional diversity. Our results demonstrate that Nav1.6 is the main Nav subunit in the somato-dendritic compartments of CA1 PCs, whereas both Nav1.6 and Nav1.2 subunits are present in AISs (17). It remains to be determined whether the axo-somatic difference in the subunit composition of Nav channels is solely responsible for the reported differences in activation threshold or whether additional differential regulation of axonal vs. somatic Nav channels also exists. Irrespective of that, our data support the view that the large axonal Nav channel density is primarily responsible for the fact that the axon has the lowest voltage threshold for generating action potentials (32). 


\section{Supplementary Material}

Refer to Web version on PubMed Central for supplementary material.

\section{Acknowledgments}

AL is the recipient of a János Bolyai Scholarship of the Hungarian Academy of Sciences. ZN is the recipient of a European Young Investigator Award (www.esf.org/euryi) and Wellcome Trust Project and Equipment grants. We would like to thank Dóra Rónaszéki and Éva Dobai for their excellent technical help; Prof. Werner Sieghart and Prof. Jean-Marc Fritschy for antibodies to the GABAA receptors; and M. Eyre and M. Farrant for their comments on the manuscript.

\section{References}

1. Losonczy A, Makara JK, Magee JC. Nature. Mar 27.2008 452:436. [PubMed: 18368112]

2. Larkum ME, Waters J, Sakmann B, Helmchen F. J Neurosci. Aug 22.2007 27:8999. [PubMed: 17715337]

3. Larkum ME, Nevian T, Sandler M, Polsky A, Schiller J. Science. Aug 7.2009 325:756. [PubMed: 19661433]

4. Kamondi A, Acsady L, Buzsaki G. J Neurosci. 1998; 18:3919. [PubMed: 9570819]

5. Losonczy A, Magee JC. Neuron. Apr 20.2006 50:291. [PubMed: 16630839]

6. Gasparini S, Magee JC. The Journal of neuroscience. Feb 15.2006 26:2088. [PubMed: 16481442]

7. Migliore M, Shepherd GM. Nat Rev Neurosci. 2002; 3:362. [PubMed: 11988775]

8. Johnston D, Magee JC, Colbert CM, Cristie BR. Annu Rev Neurosci. 1996; 19:165. [PubMed: 8833440]

9. Magee J, Hoffman D, Colbert C, Johnston D. Annu Rev Physiol. 1998; 60:327. [PubMed: 9558467]

10. Stuart G, Spruston N, Sakmann B, Hausser M. Trends Neurosci. 1997; 20:125. [PubMed: 9061867]

11. Colbert CM, Magee JC, Hoffman DA, Johnston D. The Journal of neuroscience. Sep 1.1997 17:6512. [PubMed: 9254663]

12. Gasparini S, Magee JC. The Journal of physiology. Jun 15.2002 541:665. [PubMed: 12068031]

13. Xu J, Kang N, Jiang L, Nedergaard M, Kang J. The Journal of neuroscience. Feb 16.2005 25:1750. [PubMed: 15716411]

14. Colbert CM, Johnston D. Journal of neurophysiology. Jan.1998 79:491. [PubMed: 9425219]

15. Beckh S, Noda M, Lubbert H, Numa S. The EMBO journal. Dec 1.1989 8:3611. [PubMed: 2555170]

16. Trimmer JS, Rhodes KJ. Annu Rev Physiol. 2004; 66:477. [PubMed: 14977411]

17. Hu W, et al. Nat Neurosci. Aug.2009 12:996. [PubMed: 19633666]

18. Lorincz A, Nusser Z. The Journal of neuroscience. Dec 31.2008 28:14329. [PubMed: 19118165]

19. Ogiwara I, et al. J Neurosci. May 30.2007 27:5903. [PubMed: 17537961]

20. Boiko T, et al. J Neurosci. Mar 15.2003 23:2306. [PubMed: 12657689]

21. Van Wart A, Trimmer JS, Matthews G. J Comp Neurol. Jan 10.2007 500:339. [PubMed: 17111377]

22. Gong B, Rhodes KJ, Bekele-Arcuri Z, Trimmer JS. J Comp Neurol. Sep 20.1999 412:342. [PubMed: 10441760]

23. Fujimoto K. J Cell Sci. 1995; 108:3443. [PubMed: 8586656]

24. Rash JE, Yasumura T, Hudson CS, Agre P, Nielsen S. Proc Natl Acad Sci U S A. 1998; 95:11981. [PubMed: 9751776]

25. Masugi-Tokita M, Shigemoto R. Curr Opin Neurobiol. Jun.2007 17:387. [PubMed: 17499496]

26. Lorincz A, Nusser Z. J Neurosci. Sep 10.2008 28:9083. [PubMed: 18784286]

27. Fritschy J-M, Weinmann O, Wenzel A, Benke D. J Comp Neurol. 1998; 390:194. [PubMed: 9453664]

28. Watanabe M, et al. Eur J Neurosci. 1998; 10:478. [PubMed: 9749710] 
29. Kole MH, et al. Nature neuroscience. Feb.2008 11:178.

30. Tanaka J, et al. J Neurosci. 2005; 25:799. [PubMed: 15673659]

31. Nusser Z, et al. Neuron. 1998; 21:545. [PubMed: 9768841]

32. Kole MH, Stuart GJ. Nature neuroscience. Nov.2008 11:1253. 

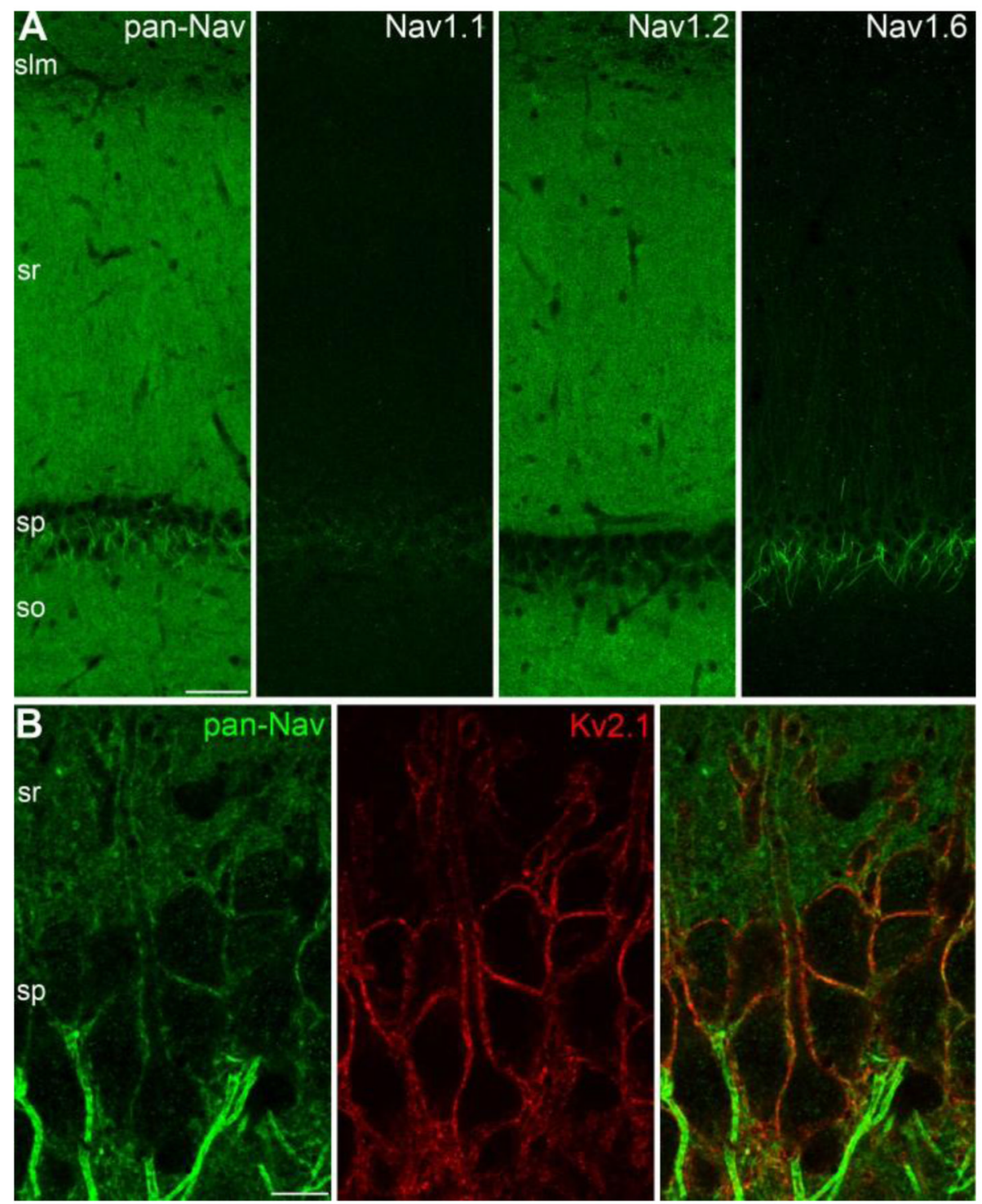

Fig. 1.

Somato-dendritic localization of voltage-gated sodium channels. (A) Immunofluorescence localization of the pan-Nav, Nav1.1, Nav1.2 and Nav1.6 subunits in the CA1 area. (B) Double immunofluorescence reaction shows weak pan-Nav immunolabeling along the Kv2.1 subunit immunoreactive somato-dendritic plasma membrane of CA1 PCs. Note the much higher labeling intensity of the AISs. slm: stratum lacunosum-moleculare, sr: stratum radiatum, sp: stratum pyramidale, so: stratum oriens; Scale bars: $\mathbf{A}, 100 \mu \mathrm{m} ; \mathbf{B}, 10 \mu \mathrm{m}$. 

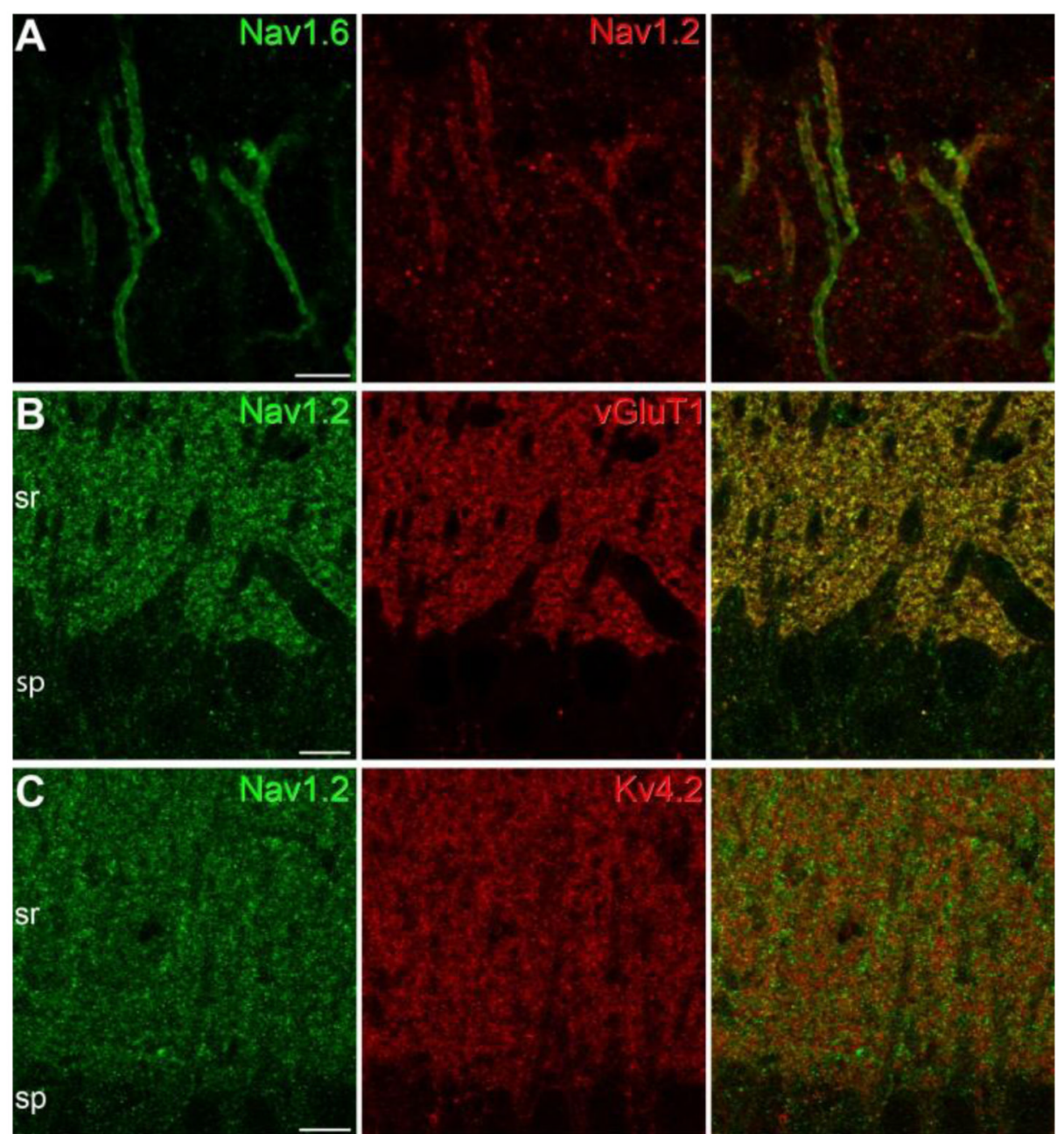

D

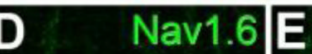

Sr
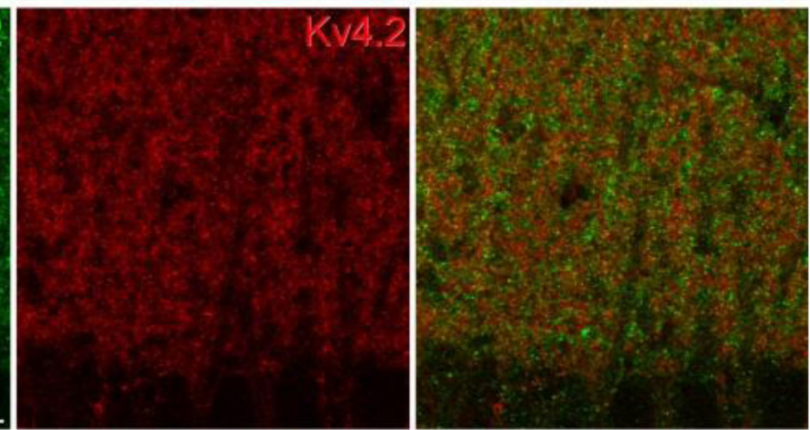

sp
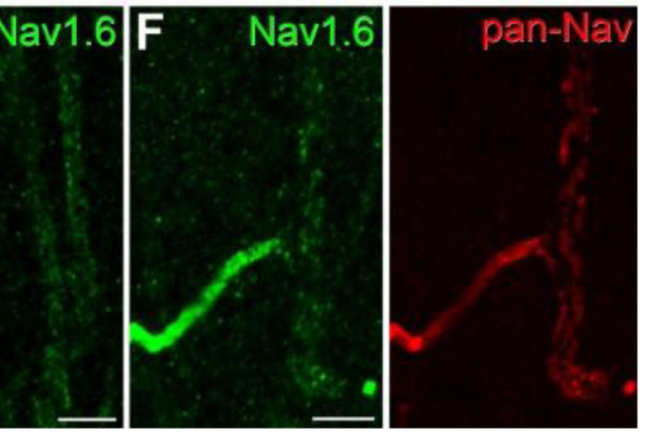

Fig. 2.

Immunofluorescence localization of the Nav1.2 and Nav1.6 subunits in CA1 PCs. (A) A double immunofluorescence reaction demonstrates the colocalization of the Nav1.6 and Nav1.2 subunits in the AISs of CA1 PCs. Immunolabeling for the Nav1.2 subunit is confined to the proximal part of the AISs. (B-C) Immunofluorescence double labeling experiments reveal that the majority of the Nav1.2 subunit immunolabeling in stratum radiatum (sr) co-localizes with the presynaptic marker vGluT1 (B), but does not co-localize with the Kv4.2 subunit $(\mathrm{C})$, a $\mathrm{K}^{+}$channel subunit that is known to be present in PC dendritic shafts and spines. (D-E) An immunofluorescence reaction for the Nav1.6 subunit in the strata pyramidale (sp) and proximal radiatum ( $\mathrm{sr}$ ) of the CA1 area demonstrates weak 
plasma membrane-like labeling of PC proximal apical dendrites. Note the much higher labeling intensity of the AISs. (F) A double immunofluorescence reaction in the stratum radiatum shows the colocalization of pan-Nav (red) and the Nav1.6 subunit (green) in a strongly immunopositive AIS and its parent apical dendrite. Scale bars: A, E, F, $5 \mu \mathrm{m}$; B-D, $10 \mu \mathrm{m}$. 


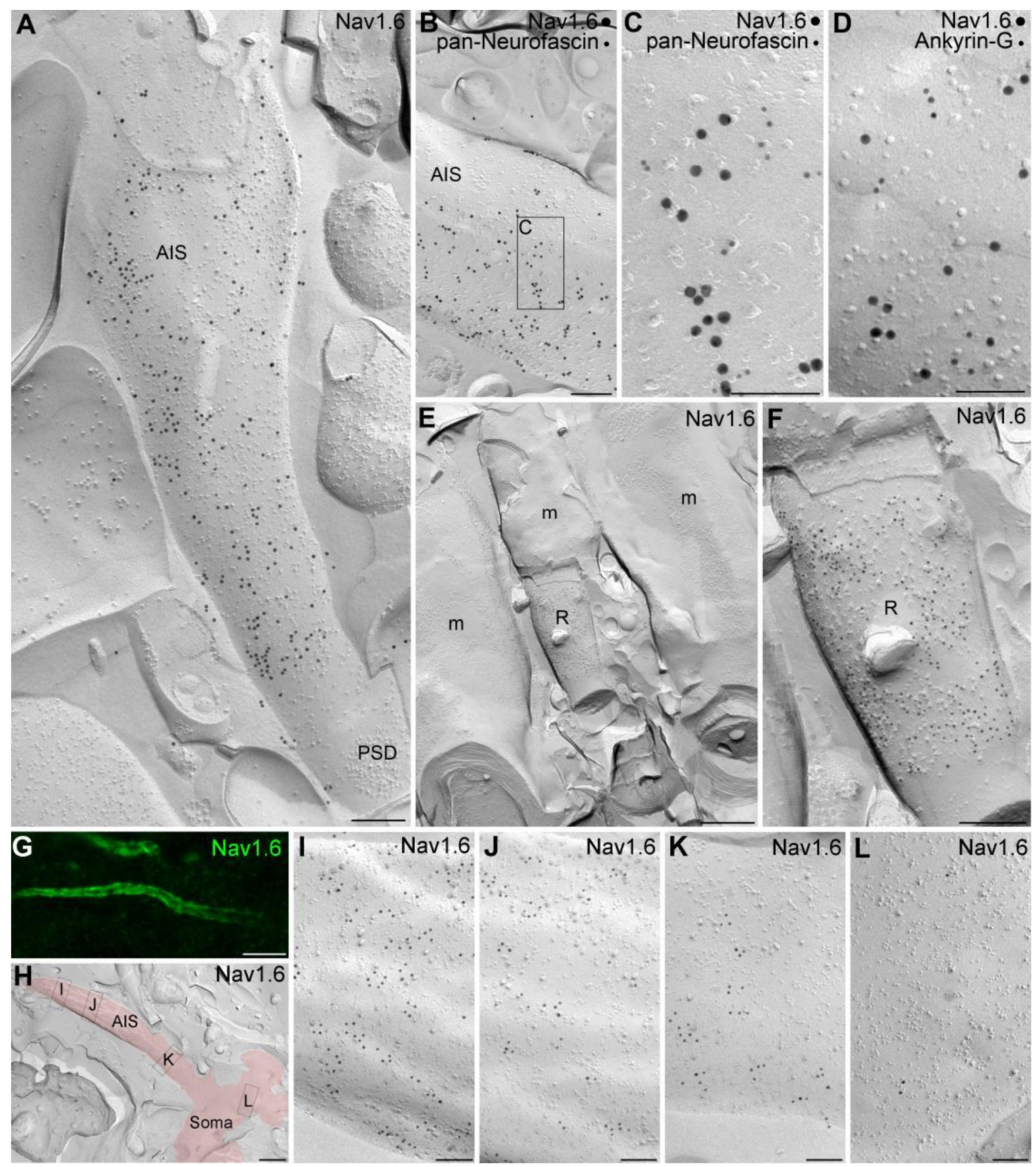

Fig. 3.

High resolution immunogold localization of the Nav1.6 subunit in AISs and nodes of Ranvier of CA1 PCs. (A) A high density of gold particles labeling the Nav1.6 subunit is found on the P-face of an AIS in the stratum pyramidale. Note that the immunogold particles avoided the area of the postsynaptic density (PSD) of an axo-axonic synapse. (B) An AIS is co-labeled for the Nav1.6 subunit (15 nm gold) and the AIS marker pan-Neurofascin (10 nm gold). (C) A high magnification view of the boxed area shown in B. (D) High magnification image of the P-face of an AIS labeled for the Nav1.6 subunit (15 nm gold) and Ankyrin-G (10 nm gold). (E) A low magnification image shows a node of Ranvier (R) of a myelinated 
axon (m) in the alveus. (F) A high magnification view of the node of Ranvier shown in $\mathbf{E}$. The P-face of the node of Ranvier membrane contains a high density of gold particles labeling the Nav1.6 subunit. Note the lack of labeling over the myelin (E and F). (G) A gradual increase in the intensity of Nav1.6 immunofluorescence is found along the proximodistal axis of CA1 PC AISs. (H) A low magnification image of a replica shows a fragment of a somatic plasma membrane and an emerging AIS (red). (I-L) High magnification images of the boxed areas shown in panel H. Images taken of the soma (L) and of the AIS at various distances from the soma (I-K) demonstrate an increase in the density of gold particles labeling the Nav1.6 subunit along the proximo-distal axis of the AIS (I). Scale bars: A, B, F, $200 \mathrm{~nm} ; \mathbf{C}, \mathbf{D}, \mathbf{I}-\mathbf{L}, 100 \mathrm{~nm} ; \mathbf{E}, 500 \mathrm{~nm} ; \mathbf{G}, 5 \mu \mathrm{m} ; \mathbf{H}, 1 \mu \mathrm{m}$. 


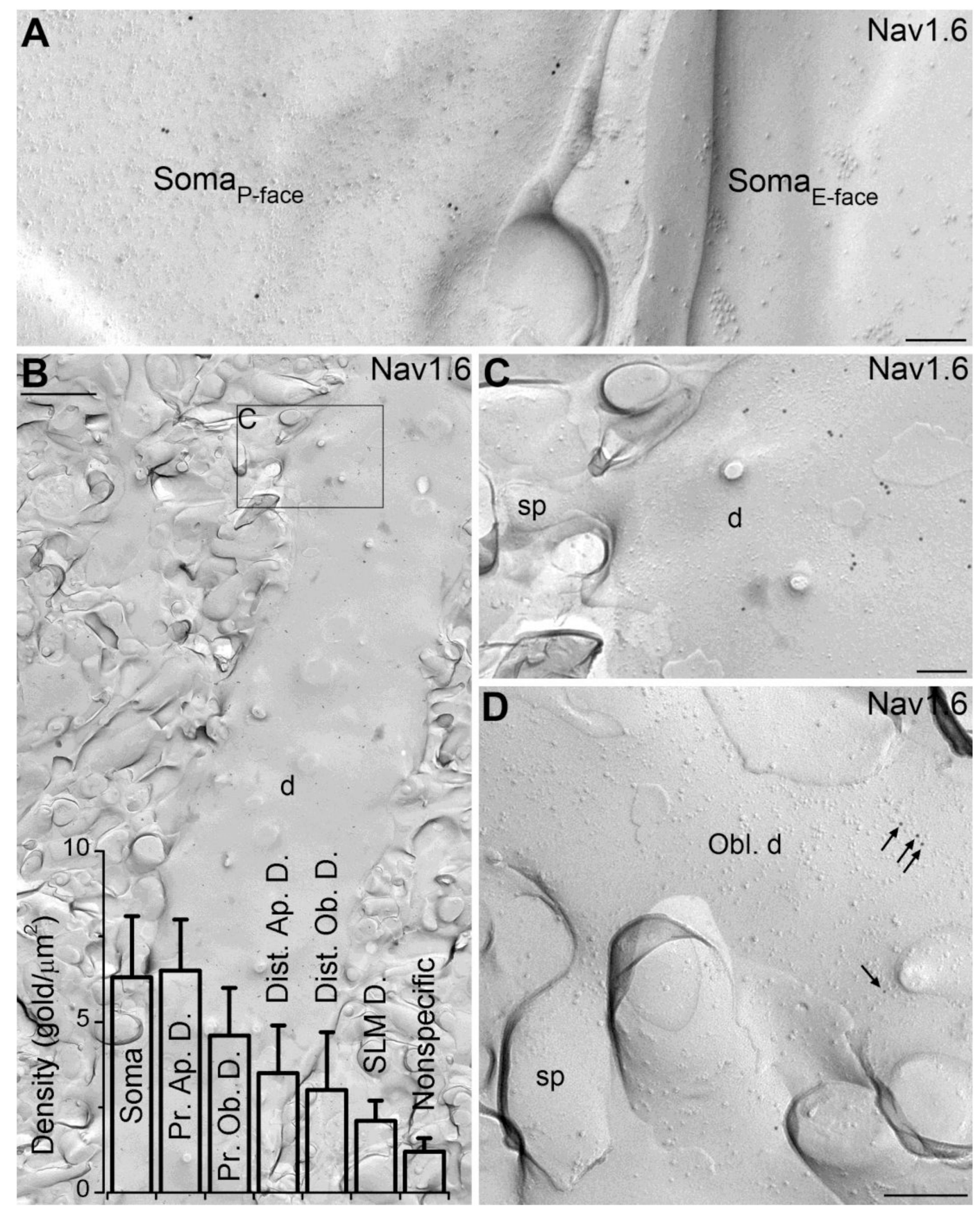

Fig. 4.

Somato-dendritic localization of the Nav1.6 subunit in CA1 PCs using SDS-FRL. (A) Low density of gold particles labeling the Nav1.6 subunit is found on P-face of the somatic plasma membrane of a CA1 PC. No immunolabeling is seen on the E-face of a neighboring PC soma. (B) Low magnification image of the P-face of a thick, spiny apical dendrite (d) of a CA1 PC in the proximal stratum radiatum labeled for the Nav1.6 subunit. Bar graphs show the densities of gold particles (mean $\pm \mathrm{SD}$ ) in somato-dendritic subcellular compartments of CA1 PCs, some of which differ significantly from the nonspecific labeling (ANOVA: $p<$ 0.001; Dunnett's post hoc test: soma, prox. apic. dendr., prox. obl. dendr., dist. apic. dendr.: $\mathrm{p}<0.05 ; \mathrm{n}=5$ rats). (C) A high magnification image of the boxed area shown in B. Gold 
particles labeling the Nav1.6 subunit are distributed on the dendritic shaft (d), but avoid the dendritic spine (sp). (D) The P-face of a spiny oblique dendrite (Obl. d) in the proximal stratum radiatum contains a low density of gold particles for the Nav1.6 subunit (arrows), with no particle in the spine (sp). Scale bars: $\mathbf{B}, 1 \mu \mathrm{m} ; \mathbf{A}, \mathbf{C}, \mathbf{D}, 200 \mathrm{~nm}$. 